\title{
STEADY STATE ANALYSIS OF CHUA'S CIRCUIT WITH RLCG TRANSMISSION LINE
}

\author{
J. Bonet-Dalmau, P. Palà-Schönwälder and F. del Águila-López \\ UPC-Department of Signal Theory and Communications \\ Avda. Bases de Manresa 61-73, Manresa 08240, Spain \\ bonet@tsc.upc.es
}

\begin{abstract}
In this paper we present a new technique to compute the steady state response of nonlinear autonomous circuits with RLCG transmission lines. Using multipoint Padé approximants, instead of the commonly used expansions around $s=0$ or $s \rightarrow \infty$, accurate, low-order lumped equivalent circuits of the characteristic impedance and the exponential propagation function are obtained in an explicit way. Then, with the temporal discretization of the equations that describe the transformed circuit, we obtain a nonlinear algebraic formulation where the unknowns to be determined are the samples of the variables directly in the steady state, along with the oscillation period, the main unknown in autonomous circuits. An efficient scheme to build the Jacobian matrix with exact partial derivatives with respect to the oscillation period and with respect to the samples of the unknowns is obtained. Steady state solutions of the Chua's circuit with RLCG transmission line are computed for selected circuit parameters.
\end{abstract}

\section{INTRODUCTION}

Several techniques have been developed to determine the steady state response of nonlinear autonomous circuits. Shooting methods, developed in the time domain, try to determine the circuit initial conditions that make zero the transient response. In circuits, which incorporate time delay, these conditions must be determined with a length equal to the maximum time delay in the circuit. In autonomous circuits, the $a$ priori ignorance of the oscillation period is an added difficulty. Methods developed in the frequency domain take advantage of the circuit division into a linear and a nonlinear part to efficiently solve the linear part. However, the nonlinear part is generally best evaluated in the time domain, which makes it necessary to take successive transformations between the two domains.

Coming to the main point of this paper, the discrete time approach (DTA) to analyze lumped nonlinear autonomous circuits proposed in [1] has been recently extended to nonlinear autonomous circuits with ideal transmission lines [2]. The method is based on the formulation of the steady state equations that describe the circuit in the time domain, without any additional transformation. After discretizing these equations, approximating the derivatives and the time delays by means of a linear combination of the samples of the discretized variables, we get an equivalent formulation of the problem in matrix form. The partial derivatives of the resulting equations with respect to the samples of the circuit variables to be determined and the oscillation period, the main unknown in autonomous circuits, are obtained in an exact analytic form, allowing the efficient implementation of globally convergent resolution techniques based on modifications of Newton's method [3]. The purpose of this paper is to extend this technique to allow the inclusion of RLCG transmission lines.

Some of the methods proposed to analyze circuits with lossy and dispersive transmission lines are based on the calculation of the impulse response of the transmission line. Then, convolution techniques are applied to determine the response until the transient dies out. These algorithms are inefficient when the transient decays very slowly compared with the period of the steady state response, resulting in excessive computing time.

To overcome this difficulty, Chang [4] proposes an approximation of the characteristic impedance

$$
Z_{0}(s)=\sqrt{\frac{R_{d}+s L_{d}}{G_{d}+s C_{d}}}
$$

and the exponential propagation function

$$
F(s)=e^{-l \sqrt{\left(R_{d}+\Delta L_{d}\right)\left(C_{d}+s C_{d}\right)}}
$$

using a lumped circuit. With this approach, the resulting circuit can be analyzed using conventional techniques. However, Alonso et al. [5] showed that the order of the lumped equivalent circuit of the exponential propagation function can be dramatically reduced using an ideal transmission line to model the major part of the physical delay. As a result, it is much more efficient to express the propagation function as

$$
F(s)=F_{c}(s) e^{-s t},
$$

with $F_{c}$ the irrational function to be approximated using a lumped circuit and

$$
e^{-s t}=e^{-s l \sqrt{L_{d} C_{d}}}
$$

the delay of and ideal transmission line. This idea is also used in [6].

There are different ways to approximate the functions $Z_{0}$ and $F_{c}$. The approach proposed in [5] consist in finding an approximate rational function using an iterative minimization algorithm. Once the 
rational function has been obtained, conventional techniques to synthesize the equivalent circuit are used. The explicit method to approximate $Z_{0}$ and $F_{c}$ described in [4] makes use of the Padé approximation at $s=0$ or $s \rightarrow \infty$ depending on the parameter values $\mathrm{R}, \mathrm{L}, \mathrm{C}$ and $\mathrm{G}$ of the transmission line. However, errors are unnecessarily high at the opposite frequency $s \rightarrow \infty$ or $s=0$, respectively

To overcome this difficulty, in this paper multipoint Padé approximants [7] are used, approximating these functions over the whole frequency axis. Once the approximations are obtained, they are directly included in the DTA formulation without having to explicitly synthesize the corresponding circuit. At the same time, in [8] it has been showed that the procedure to compute the multipoint Padé approximants allows us to efficiently include frequency dependent resistance parameters, including $k \sqrt{ } s$ terms, accounting for skin-effect losses.

\section{APPLICATION TO CHUA'S CIRCUIT WITH RLCG TRANSMISSION LINE}

The described technique has been applied to the determination of the steady state of the control variable $v$ in Chua's circuit with transmission line shown in Fig. 1. The values of the circuit parameters, the same as in [6], are $Z_{L}=0 \Omega, R_{d}=0.55 \Omega / \mathrm{m}, L_{l}=0.2375 \mu \mathrm{H} / \mathrm{m}$, $G_{l}=0.1 \mathrm{mS} / \mathrm{m} \quad C_{l l}=95 \mathrm{pF} / \mathrm{m}, \quad l=3 \mathrm{~m}, \quad C=1 \mathrm{pF} \quad$ and $R=1.83 \mathrm{k} \Omega$. The $i-v$ characteristic of the nonlinearity is depicted in Fig. 2 with $m_{0}=-0.9 \mathrm{mS}, m_{l}=-0.5 \mathrm{mS}$ and $v_{a}=1 \mathrm{~V}$. For computation purposes these values have been normalized with $R_{0}=1 \mathrm{k} \Omega$ and $T_{0}=60 \mathrm{~ns}$.

The RLCG transmission line of this circuit is equivalent to the two-port shown in Fig. 3, where

$$
\begin{aligned}
& E_{1}(s)=F_{c}(s) e^{-s t}\left(2 V_{2}-E_{2}\right), \\
& E_{2}(s)=F_{c}(s) e^{-s t}\left(2 V_{1}-E_{1}\right) .
\end{aligned}
$$

For the selected circuit parameter $Z_{L}=0 \Omega, v_{1}=0 \mathrm{~V}$, the output port of the RLCG line can be characterized as a one-port with equivalent impedance

$$
Z\left(s, e^{-2 s \tau}\right)=Z_{0}(s) \frac{1+F_{c}^{2}(s) e^{-2 s \tau}}{1-F_{c}^{2}(s) e^{-2 s \tau}}
$$

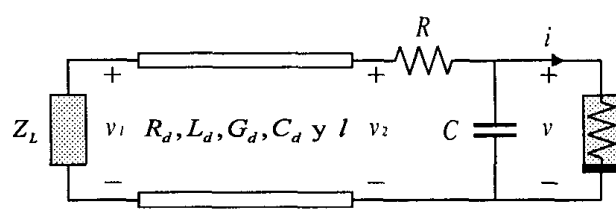

Fig. 1. Chua's circuit with transmission line [6].

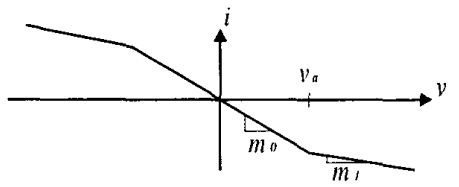

Fig. 2. The $i-v$ characteristic of the piecewise linear resistor of Fig. 1.
With this expression, and substituting the nonlinearity by a controlled source, the circuit of Fig. 1 is transformed into the circuit of Fig. 4. Following DTA method described in [2], the first step consists in obtaining the equilibrium equations where the control variables of the nonlinearities are expressed as a function of the controlled and bias sources of the circuit. The equilibrium equation of the considered circuit is

$$
A(s) V(s)+B(s) I(V)=0,
$$

with

$$
\begin{gathered}
A(s)=1+\left(R+Z_{0}\right) C s+\left(1+\left(R-Z_{0}\right) C s\right) F_{c}^{2} e^{-2 s \tau}, \\
B(s)=\left(R+Z_{0}\right)+\left(R-Z_{0}\right) F_{c}^{2} e^{-2 s \tau} .
\end{gathered}
$$

However, the DTA method can only deal with $A$ and $B$ being bivariate polynomials of the type

$$
P(s)=\sum_{i=0}^{n} \sum_{k=0}^{2} p_{i k} s^{i} e^{-s k \tau},
$$

where $n$ is the order of the lumped linear subcircuit. As $Z_{0}$ and $F_{c}$ are irrational functions, an approximation with the following rational functions is essential to apply the DTA method.

$$
\begin{gathered}
Z_{0}(s) \cong \frac{Z_{a}(s)}{Z_{b}(s)}=\frac{\sum_{i=0}^{n_{Z_{0}}} a_{i} s^{i}}{\sum_{j=0}^{n_{Z_{0}}} b_{j} s^{j}}, \\
F_{c}(s) \cong \frac{F_{a}(s)}{F_{b}(s)}=\frac{\sum_{i=0}^{n_{k_{k}}} c_{i} s^{i}}{\sum_{j=0}^{n_{t_{k}}} d_{j} s^{j}} .
\end{gathered}
$$

Choosing $n_{z 0}=1$ and $n_{F_{i}}=1$ there are four coefficients and one degree of freedom, so it is possible to fix three conditions of coincidence between the original and the approximated rational function. To approximate over the whole axis frequency the best results are obtained fitting the coincidence of the values of the original and the rational function at both ends of the axis frequency and at a point about which the functions are symmetric, that is

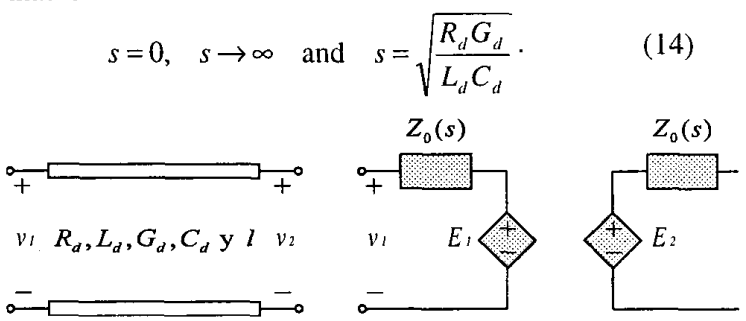

Fig. 3. Equivalent two-port of an RLCG line.

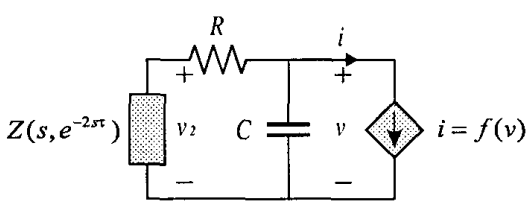

Fig. 4. Substitution of the RLCG line by its equivalent impedance and the nonlinearity by a controlled source. 
Thus, substituting the first order approximation of the characteristic impedance

$$
Z_{0}(s) \cong \frac{Z_{a}(s)}{Z_{b}(s)}=\frac{a_{0}+a_{1} s}{b_{0}+b_{1} s}
$$

and the propagation function

$$
F(s)=F_{c}(s) e^{-s \tau} \cong \frac{F_{a}(s)}{F_{b}(s)} e^{-s \tau}=\frac{c_{0}+c_{1} s}{d_{0}+d_{1} s} e^{-s \tau}
$$

in (9) and (10), and reorganizing the terms, the equilibrium equation

$$
D(s) V(s)+E(s) I(V)=0
$$

is obtained, with

$$
\begin{gathered}
D(s)=\left(Z_{b}+\left(R Z_{b}+Z_{a}\right) C s\right) F_{b}^{2}+ \\
\left(Z_{b}+\left(R Z_{b}-Z_{a}\right) C s\right) F_{a}^{2} e^{-2 s \tau} \\
E(s)=\left(R Z_{b}+Z_{a}\right) F_{b}^{2}+\left(R Z_{b}-Z_{a}\right) F_{a}^{2} e^{-2 s \tau} .
\end{gathered}
$$

It should be noted that the approximations (15) and (16) need a number of dynamic elements equal to $n_{z 0}$ and $n_{F}$ respectively. Thus, $n$, the order of the lumped linear subcircuit which appeared in (11), takes the value

$$
n=\left(n_{z_{0}}+2 n_{r_{r}}\right)+1=4
$$
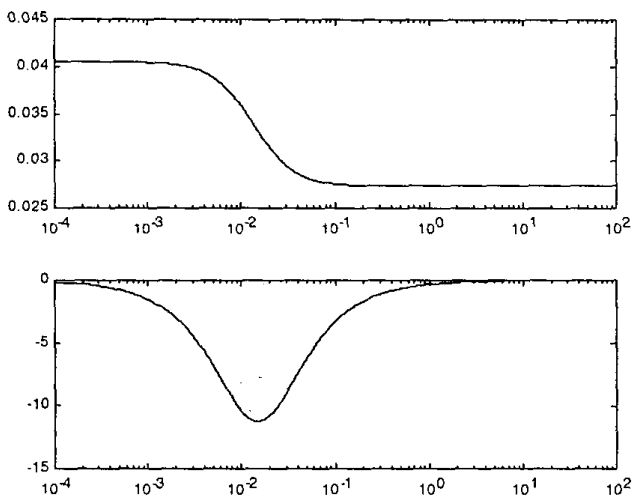

Fig. 5. Modulus (above) and phase in degrees (bellow) of $Z_{0}$ as a function of normalized frequency.
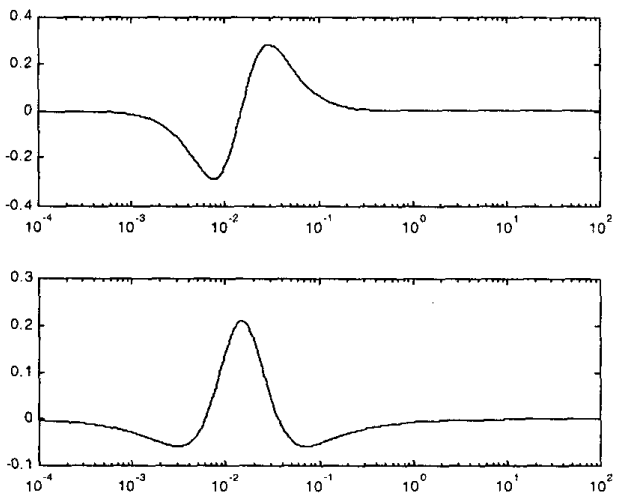

Fig. 6. Relative error (\%) in the modulus approximation (above) and absolute error degrees in the phase approximation (bellow) of $Z_{0}$ with $n_{z 0}=1$. after the approximation of the RLCG transmission line (note that using an ideal transmission line we would obtain $n=1$ ).

Once $D$ and $E$ are polynomials of the type indicated in (11), the DTA method can be applied with no limitation. The expression (17) translated to the time domain is a nonlinear difference differential equation. This equation is discretized, so that each one of the operators derivative and delay are approximated as a linear combination of $g+1$ samples of the variables acted upon by the operator, being $g$ the order of the discretization. The Gear based criterion used to obtain these approximations is detailed in [2]. Summarizing the method, it transforms each variable into a periodic vector of $N$ samples, the operator $s$ into a matrix $\mathbf{P}_{10}$ of dimensions $N \mathrm{x} N$ and the operator $e^{-2 s \tau}$ into a matrix $\mathbf{P}_{02}$ of dimensions $N \mathrm{x} N$. So, a nonlinear algebraic equation is obtained, constructed with only two different matrices. These matrices are circulant and sparse, since only exist $g+1$ nonzero elements in each row of the matrix, properties that can be exploited to reduce the computation time.
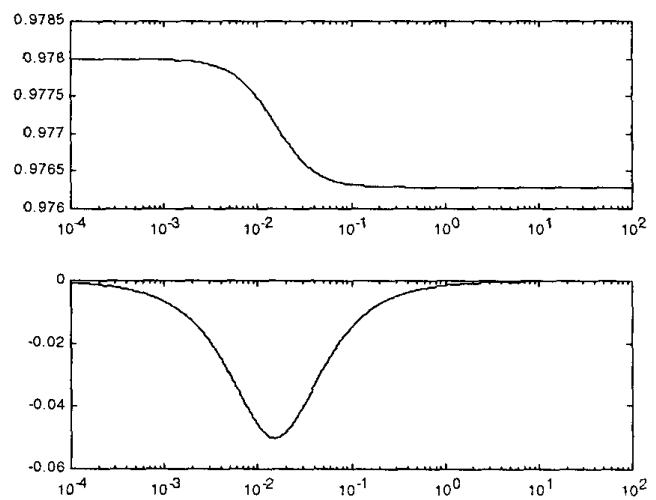

Fig. 7. Modulus (above) and phase in degrees (bellow) of $F_{c}$ as a function of normalized frequency.
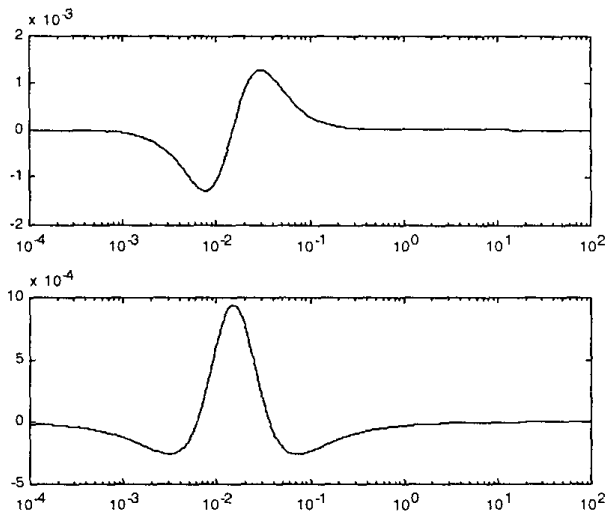

Fig. 8. Relative error (\%) in the modulus approximation (above) and absolute error degrees in the phase approximation (bellow) of $F_{c}$ with $n_{F c}=1$. 


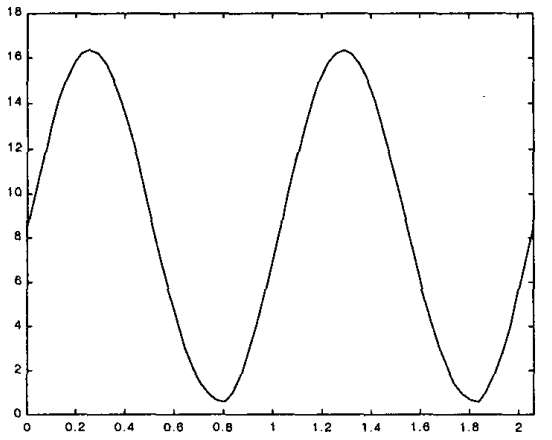

Fig. 9. Two periods of the waveform $v$, obtained using the described algorithm.

The Jacobian matrix of the nonlinear system of equations - which includes de partial derivatives with respect to the oscillation period- can be analytically computed, allowing the efficient implementation of globally convergent resolution techniques based on modifications of Newton methods.

\section{SIMULATION RESULTS}

The simulation results are as follows. The functions $Z_{0}$ and $F_{c}$, whose modulus and phase are represented in Fig. 5 and Fig. 7, have been approximated on $n_{z 0}=1$ and $n_{F c}=1$ with the error represented in Fig. 6 and Fig. 8. The results using higher values of $n_{z 0}$ and $n_{F c}$ do not differ from the ones obtained next. A second-order Gear discretization has been used to approximate the derivative and delay operators. The initialization of the iterative process has been made with $N=32$ samples of a sinusoidal signal, obtaining the waveform depicted in Fig. 9 and its corresponding phase plane depicted in Fig. 10. The results show excellent agreement with those obtained using the technique in [6], without having to compute the response until the transient dies out, i.e. with a much lower computational cost. This agreement also shows that the multipoint Padé approximants offers an equivalent performance with lower approximant order.

\section{CONCLUSIONS}

A new method to determine the steady state response of nonlinear autonomous circuits with RLCG transmission lines has been presented. To overcome the difficulty of analyzing lossy and dispersive transmission lines, multipoint Padé approximants are used in the first stage. Thus, the original circuit is transformed in a lumped circuit with ideal transmission lines. In the next stage, the equations that describe the circuit are discretized in the time domain, transforming the initial problem, the solution of a nonlinear difference differential system of equations, into the solution of a nonlinear algebraic system of equations.

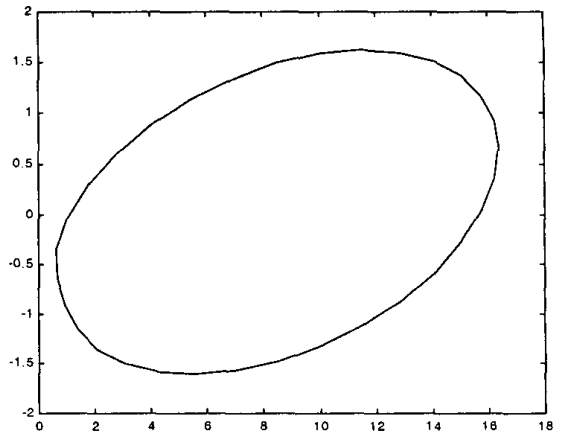

Fig. 10. Phase plane, $v_{2}-v$, of the solution depicted in Fig. 9.

To efficiently solve the resulting system of nonlinear equations, globally convergent algorithms based on Newton's method have been implemented. The exact analytic computation of the required partial derivatives can be easily carried out.

To validate the method, it has been applied to the determination of the steady state response of Chua's circuit with transmission line in one of its periodic windows. The results coincide with those obtained using integration techniques, with the advantage of not having to compute the response until the transient dies out.

\section{REFERENCES}

[1] P. Palà-Schönwälder and J. M. Miró-Sans, "A discretetime approach to the steady-state analysis and optimization of non-linear autonomous circuits," Int. $J$. Circuit Theory Appl., vol. 23, pp. 297-310. 1995.

[2] J. Bonet-Dalmau, P. Palà-Schönwälder and J. M. Miró-Sans, "A discrete-time approach to the steady state analysis of distributed nonlinear autonomous circuits," in Proc. IEEE ISCAS'98, 1998, vol. 3, pp. 460-464.

[3] J. E. Dennis and R. B. Schnabel, Numerical Methods for Unconstrained Optimization and Nonlinear Equations. Englewood Cliffs, NJ: Prentice-Hall, 1983.

[4] F. Y. Chang, "Waveform relaxation analysis of RLCG transmission lines," IEEE Trans. Circuits Syst., vol. 37, pp. 1394-1415, 1990

[5] J. I. Alonso, J. Borja and F. Pérez, "A universal model for lossy and dispersive transmission lines for time domain CAD of circuits," IEEE Trans. Microwave Theory Tech., vol. 40, pp. 938-946, 1992.

[6] J. Kawata, Y. Nishio and A. Ushida, "Analysis of Chua's circuit with transmission line," IEEE Trans. Circuits Syst. I, vol. 44, pp. 556-558, 1997.

[7] G. A. Baker Jr. and P. R. Graves-Morris, Padé Approximants, Part I: Basic Theory, Part Il: Extensions and Applications. London: Addison-Wessley, 1981.

[8] P. Palà and J. M. Miró, "An explicit method for modeling lossy and dispersive transmission lines," in Proc. $23 \mathrm{rd}$ European Microwave Conference, 1993, pp. 701-704. 